\title{
Sistemas mHealth para la adquisición de señales EEG
}

\author{
Cesar Fabian Reyes Manzano ${ }^{1}$, Blanca Alicia Rico Jiménez ${ }^{2}$ y Laura Ivoone Garay \\ Jiméne $z^{3}$ \\ ${ }^{1}$ Instituto Politécnico Nacional, UPIITA, SEPI, D.F., México. \\ cesarrm5@hotmail.com \\ ${ }^{2}$ Instituto Politécnico Nacional, UPIITA, informática, D.F., México. \\ bricoj@ipn.mx \\ ${ }^{3}$ Instituto Politécnico Nacional, UPIITA, SEPI, D.F., México. \\ lgaraydipn.mx
}

\begin{abstract}
Resumen. El incremento en el uso de dispositivos móviles ha generado un crecimiento de puntos de acceso, públicos y privados, esto es aprovechado por los sistemas mHealth, los cuales utilizan la infraestructura de los servicios móviles para brindar servicios de salud, realizando aplicaciones como el monitoreo de señales biológicas. Una de estas aplicaciones puede ser la medición de la señal de electroencefalografía (EEG), la cual consiste en adquirir la señal eléctrica de la corteza cerebral de forma no invasiva.

Este artículo propone un sistema capaz de obtener múltiples registros por medio de módulos con 19 sensores de señal EEG y un envío de datos usando el protocolo Wi-Fi hacia un servidor por medio de socket TCP.

El sistema desarrollado permite la realización de hasta 12 estudios en forma paralela y de manera remota, como puede ser el hogar del paciente, una clínica rural, etc., con una eficiencia del $99.21 \%$ de datos trasmitidos correctamente.
\end{abstract}

Palabras clave: electroencefalográfica, mHealth, TCP.

\section{Introducción}

La electroencefalografía consiste en medir la señal eléctrica de la corteza cerebral por medio de electrodos colocados sobre el cuero cabelludo [1]. Este es un método no invasivo que permite conocer el estado del cerebro con alta resolución temporal. El conocer el estado del cerebro es de gran ayuda para el diagnóstico de enfermedades neurológicas, sin embargo, la demanda de servicio en el sector salud y la infraestructura generada por el sector público es insuficiente para los usuarios demandantes, por lo cual, es deseable contar con sistemas que coadyuven a la atención de los pacientes optimizando la infraestructura disponible [2]. Una alternativa de solución propuesta es el uso de sistemas mHeath, los cuales utilizan la infraestructura de las redes de sistemas móviles para transmitir el flujo de información entre el paciente y el médico, sin importar la distancia a la que se encuentren [3].

En la actualidad, se han desarrollado sistemas de adquisición móvil con la finalidad de obtener señales electroencefalográficas. El tipo de aplicación del equipo condicio- 
na la resolución, frecuencia de muestreo y el tipo de tratamiento de la señal tanto a nivel analógico como digital. En las interfaces Hombre Máquina se buscan parámetros generales que permitan identificar estados de actividad neurológica para controlar procesos o desencadenar acciones. Es por esto que se da prioridad a la transmisión en tiempo real sobre la fidelidad de la información, pero sin comprometer la calidad. Uno de estos sistemas es el EPOC $®$ (Emotiv, USA), que se usa en sistemas de interface Cerebro-Computadora (BCI) y cuenta con 14 canales [4]; la colocación de los electrodos se distribuye en la zona motora, debido a que se utiliza para el control de dispositivos a partir de señales asociadas al movimiento.

En cambio, en el uso para estudios clínicos es de suma importancia mantener la mayor fidelidad posible tanto temporal como espacialmente debido a que se busca analizar, clasificar y asociar morfologías de la señal EEG a patologías clínicas, así como la interpretación de esta señal con métodos automáticos utilizando técnicas de procesamiento $[5,6,7,8]$. Para estas aplicaciones se pueden encontrar en el mercado el sistema "EEG: DSI 10/20" de la compañía QUASAR ${ }^{\mathrm{TM}}$, que cuenta con 21 electrodos [9]; y el "B-Alert X24" de la compañía “Advances Brain Monitoring” [10] con 20 electrodos. Ambos proponen la colocación de los electrodos en base al sistema 10-20, el cual es un estándar médico que ayuda a una correcta interpretación de la lectura de la señal. Todos los sistemas considerados cuentan con comunicación inalámbrica, a una computadora personal o a un dispositivo móvil, utilizando Bluetooth lo que permite la movilidad exclusivamente dentro del área de registro. Los sistemas cuentan con un software de uso específico proporcionado por el fabricante para el envío o almacenamiento de las señales adquiridas.

Los sistemas mHealth utilizan la infraestructura de los dispositivos móviles para brindar servicios de salud con la finalidad de generar una cobertura en cualquier lugar y cualquier momento [11]. Estos sistemas pueden ser usados en el monitoreo a pacientes con enfermedades crónico degenerativas, como es el caso de la Diabetes Mellitus. Un ejemplo es una aplicación para iPhone ${ }^{\circledR}$ (APPLE, USA) que ayuda a monitorear la cantidad de glucosa del paciente por medio de una conexión a un dispositivo medidor de glucosa. La aplicación informa al paciente cuando y cuanta insulina debe aplicarse [12]. Otra función relevante de los sistemas mHealth es el monitoreo a distancia de pacientes, conocida como telemetría. Estos sistemas han tenido un amplio desarrollo en el área de cardiología, entre los avances se encuentra la adquisición de la señal electrocardiográfica (ECG) por un dispositivo que la envía a PDA (asistente personal digital) por medio de Bluetooth, y la PDA a su vez la envía a un servidor por medio de una conexión a internet. La aplicación busca auxiliar en el diagnóstico de arritmias cardiacas en niños, que no pudieron ser detectadas en exámenes de corta duración [13].

Estos sistemas han evolucionado con la introducción de nuevos dispositivos móviles, como es el proyecto MTM-1, que realiza la misma función de proyecto anterior usando teléfonos inteligentes con sistema operativo Android [14]. Es importante mencionar que los nuevos dispositivos móviles cuentan con una mayor capacidad de procesamiento lo que permite realizar aplicaciones para la búsqueda y análisis previo de características de la señal ECG, reduciendo la cantidad de datos enviados y teniendo una mayor interacción con el usuario [15].

Los avances mostrados anteriormente dan las bases para plantear nuevos objetivos en los que se encuentra el medir señales más complejas que la señal ECG, como es la 
señal de electroencefalografía que cuenta con un número mayor de canales y una amplitud menor. Por otro lado, el crecimiento de sistemas de conexión a la red creada para los sistemas telefónicos, dan pauta a la posibilidad de crear dispositivos embebidos que utilicen una conexión a internet sin la necesidad de un teléfono móvil intermedio [16].

Considerando los avances en las prestaciones tanto de procesamiento, como de cobertura y memoria de los dispositivos móviles, este trabajo propone el diseño y construcción de un sistema de adquisición de señal EEG siguiendo la filosofía mHealth, es decir, realizar el registro de señal EEG por medio de sistemas móviles que envíen los datos a través de puntos de acceso Wi-Fi.

En la siguiente sección se describe el funcionamiento general del sistema, y a detalle cada uno de sus elementos. En la sección 3 se describen los dispositivos que adquieren la señal EEG para enviarla al servidor que son denominados electroencefalógrafos. En la sección 4 se describe el manejo de conexiones hacia el servidor y el almacenamiento de los datos recibidos. En la sección 5 se muestran los resultados obtenidos y la evaluación de estos. En las últimas secciones se presentan la discusión y las conclusiones.

\section{Descripción del sistema}

El módulo de adquisición de la señal EEG (electroencefalógrafo) permite el envío de la señal adquirida a través de internet por medio del protocolo Wi-Fi, con el fin de que se pueda realizar el estudio de electroencefalografía desde cualquier lugar, que cuente con un punto de acceso Wi-Fi. La señal es recibida en un servidor por la aplicación desarrollada, la cual se nombró “Alimón”. Ésta se encarga de establecer y manejar las conexiones, así como del almacenamiento de los datos recibidos. En la figura 1, el diagrama del sistema muestra tres módulos de adquisición de señal EEG que envían los datos al servidor a través de diferentes puntos de acceso. Cada uno de los elementos se describe a continuación:

- Electroencefalógrafo: Es un sistema de registro de señales electroencefalográficas que contiene un microcontrolador LM4F232H5QC (Texas ${ }^{\circledR}$, USA), el cual se encarga de tomar la lectura de los 19 sensores de electroencefalografía, digitalizarlos y enviarlos por Wi-Fi a un punto de acceso, para su transmisión a través de la red.

- Punto de acceso: Este es un enrutador que tiene la función de direccionar y controlar el flujo de los paquetes enviados a través de una red WLAN, así como asignar direcciones IP a los elementos de la red y acondicionar los datos para envío a través de internet. El enrutador usado es el modelo DIR-615 (D-Link, USA) en el que se configuraron los datos de la red (nombre de la red, seguridad WPA2, etc.) y un permiso para la utilización del puerto por el que se envían los datos (TCP4444), esto se realiza en la página de configuración del enrutador (http://192.168.0.1).

- Servidor: Es una computadora personal marca Lenovo, modelo Ideapad Y400 con procesador i7 y $6 \mathrm{~Gb}$ en RAM, con sistema operativo Fedora 19 y configurada con una IP fija. En esta computadora se ejecuta el programa "Alimón”, que se encarga 
del manejo de las conexiones de cada uno de los sistemas de registro.

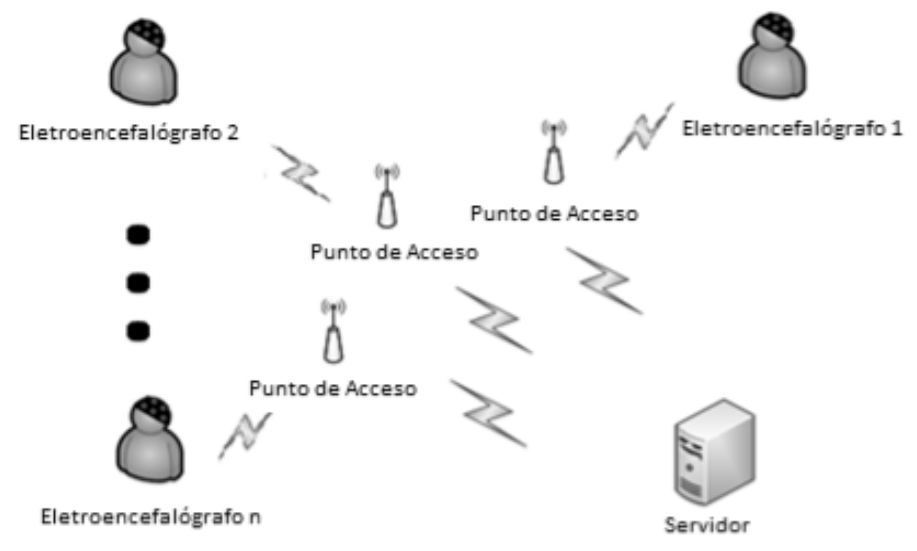

Fig. 1. Diagrama del sistema mHealth para la adquisición de la señal EEG

\section{Adquisición de datos}

El electroencefalógrafo realiza la adquisición de las señales electroencefalográficas. En la figura 2 se muestra el diagrama general del sistema. Las señales son obtenidas por medio de 19 sensores los cuales tienen 2 salidas analógicas: una correspondiente a la señal electroencefalográfica, la cual es tomada por electrodos de contacto superficial, amplificada con una ganancia de 1000 y filtrada con una banda de 0.05 a $130 \mathrm{~Hz}$; y otra salida correspondiente a la impedancia de la interface electrodo-piel. Una descripción detallada de este dispositivo se encuentra en [17].

Las señales adquiridas por los sensores son digitalizadas por un microcontrolador usando una frecuencia de muestreo de $500 \mathrm{~Hz}$ y una resolución de 12 bits, para posteriormente empaquetarlas y enviarlas por medio de un módulo Wi-Fi.

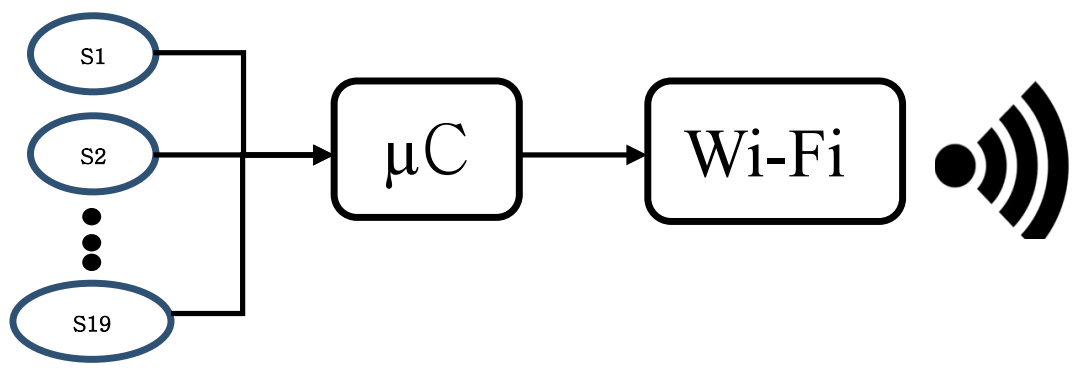

Fig. 2. Diagrama de electroencefalógrafo.

En la figura 3 se muestra el diagrama de flujo del proceso de digitalización y envío de las señales en el microcontrolador y se describe a continuación: 
- Se configura el electroencefalograma en la red WLAN correspondiente al punto de acceso.

- Se crea un socket tipo TCP en modo cliente hacia el servidor.

- Se envían los datos correspondientes al electroencefalógrafo (nombre del equipo, frecuencia de muestreo, resolución de la muestra y longitud del paquete).

- Se inicia el registro de las señales electroencefalográficas con una frecuencia de muestreo de $500 \mathrm{~Hz}$.

- Al almacenar 8 muestras de señal electroencefalográfica por canal, se realiza un muestreo de la señal de impedancia.

- Genera un paquete de datos con las muestras realizadas (8 de las señales electroencefalográficas y una de impedancia.)

- Envía el paquete por el socket TCP.

- Regresa y espera nuevamente el almacenamiento de 8 muestras.

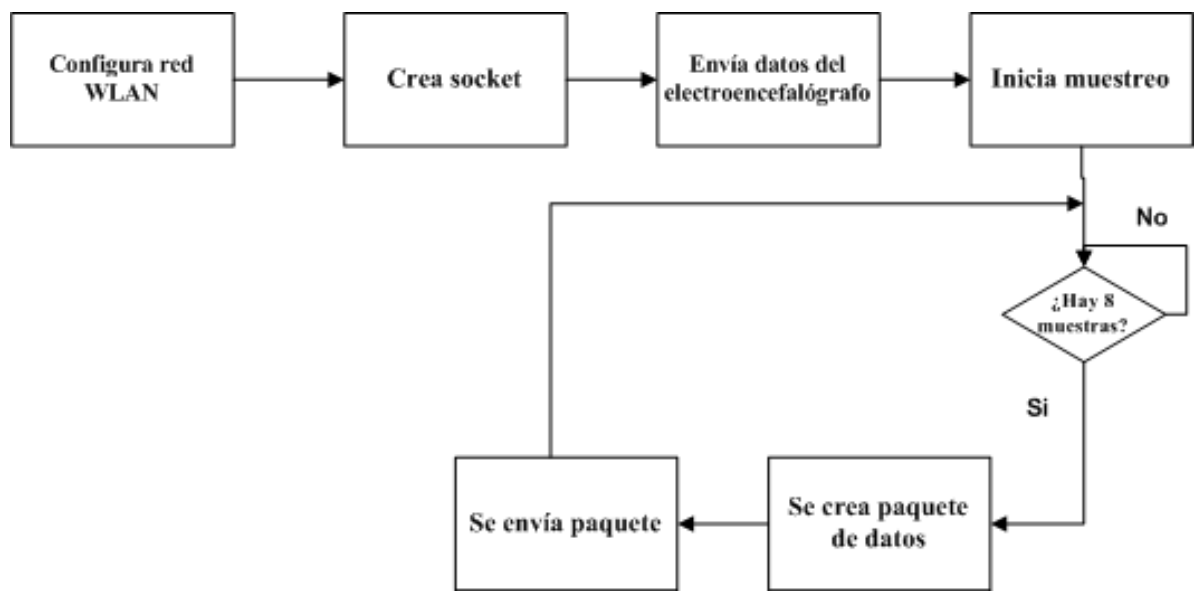

Fig. 3. Proceso de adquisición y envío de las señales electroencefalográficas y de impedancia.

Este sistema se repite para cada uno de los usuarios monitoreados, distinguiéndose únicamente por el nombre que se asigna a cada equipo para su reconocimiento por el programa "Alimón".

\section{Manejo de conexiones y almacenamiento de la señal}

Para controlar las conexiones de los dispositivos que registran desde diferentes puntos de internet, se desarrolló un programa en lenguaje Java versión 1.7.0_45 denominado "Alimón". Éste se ejecuta en el servidor y sigue el proceso mostrado en el diagrama de actividades de las figuras 4 y 5 , el cual se describe a continuación:

- Crea socket TCP en modo servidor.

- Espera la conexión de un cliente. 
- Al recibir una petición de conexión, el programa realiza la conexión y se genera un hilo de procesamiento en donde se crean los archivos necesarios para el almacenamiento de las señales.

- El hilo principal regresa a esperar una petición de conexión.

- El hilo creado, inicia un ciclo de recepción, desempaquetado y almacenamiento de la señal.

- El programa muestra los datos recibidos en 2 pantallas. Una correspondiente a las señales electroencefalográficas y otra para las señales de impedancia.

- El hilo cierra la conexión y finaliza cuando el electroencefalógrafo deja de enviar paquetes por más de 180 segundos.

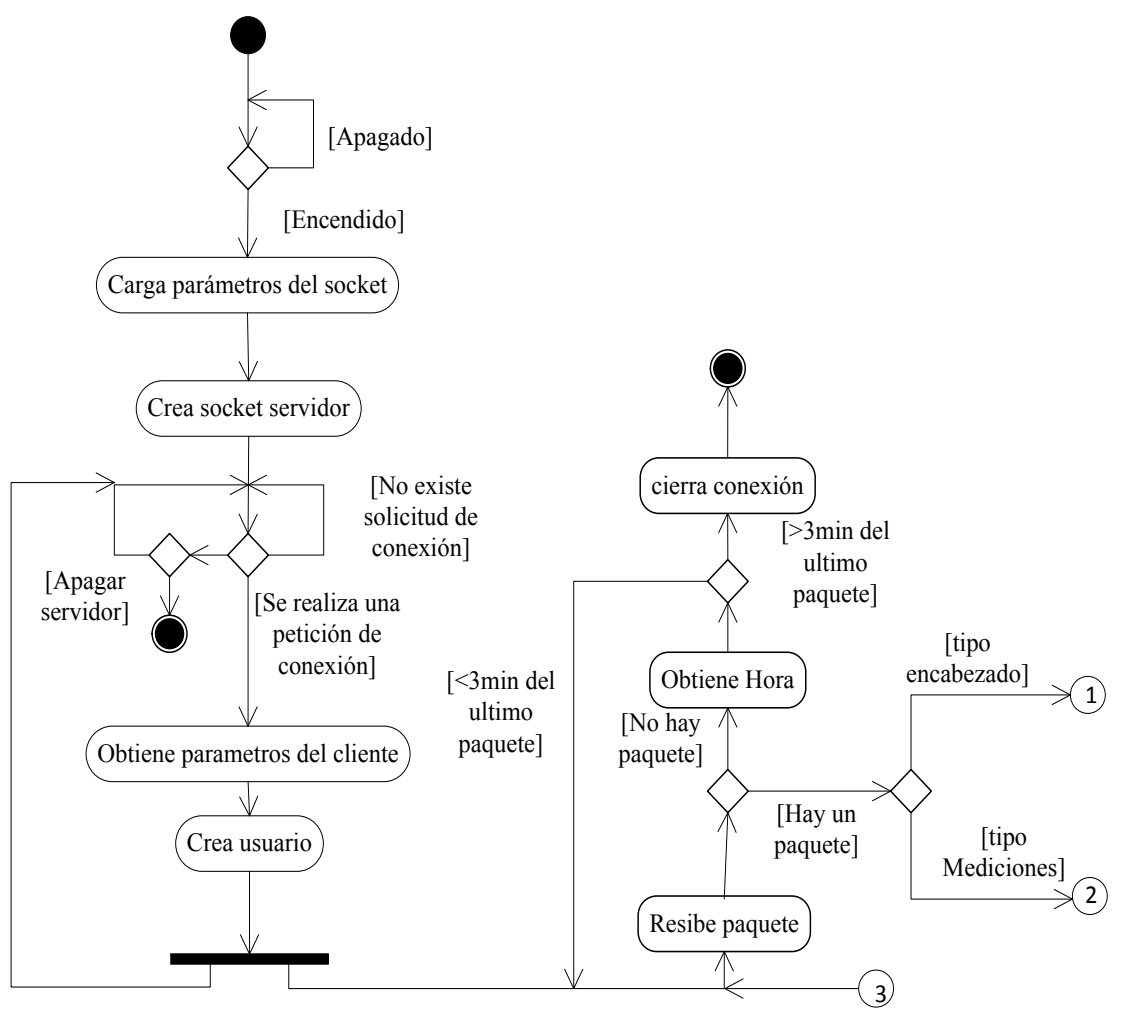

Fig. 4. Diagrama de actividades del programa "Alimón" para el manejo de conexiones y almacenamiento de la señal. 


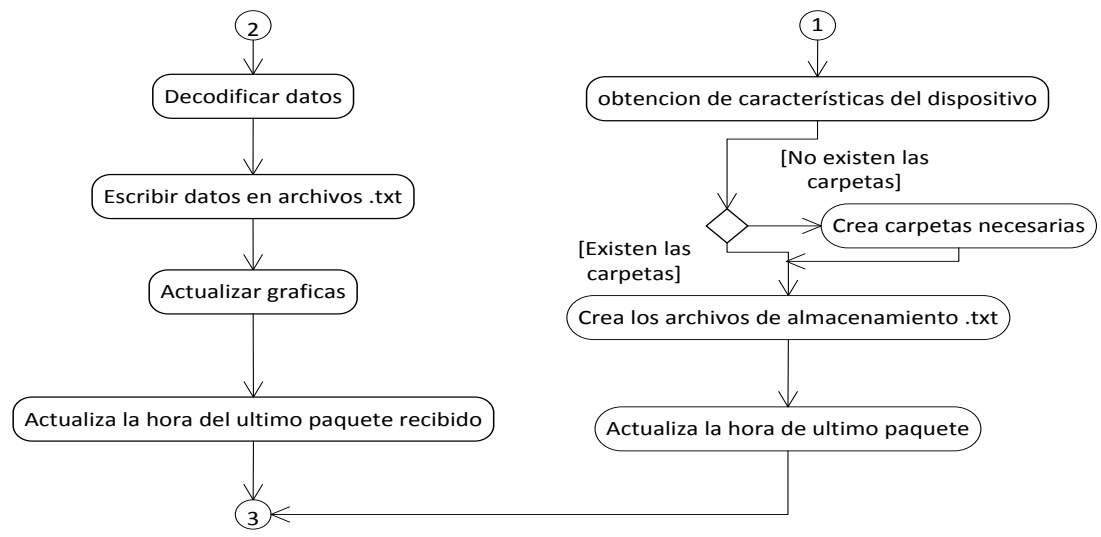

Fig. 5. Diagrama de actividades del programa “Alimón” para el manejo de conexiones y almacenamiento de la señal (continuación).

\section{Resultados y evaluación}

Se obtuvo un sistema de adquisición de señal electroencefalográfica capaz de transmitir la señal adquirida a través del protocolo Wi-Fi y enviar por medio de internet a un servidor donde se almacena y visualiza la señal. El programa para el manejo de conexiones y almacenamiento de datos es mostrado en la figura 6 , donde se observa gráficamente el estado de la conexión de los dispositivos y las señales obtenidas para cada uno de ellos. La información de las señales obtenidas se guardó en formato texto con la finalidad de que se pueda analizar posteriormente mediante cualquier paquetería comercial o de uso libre.

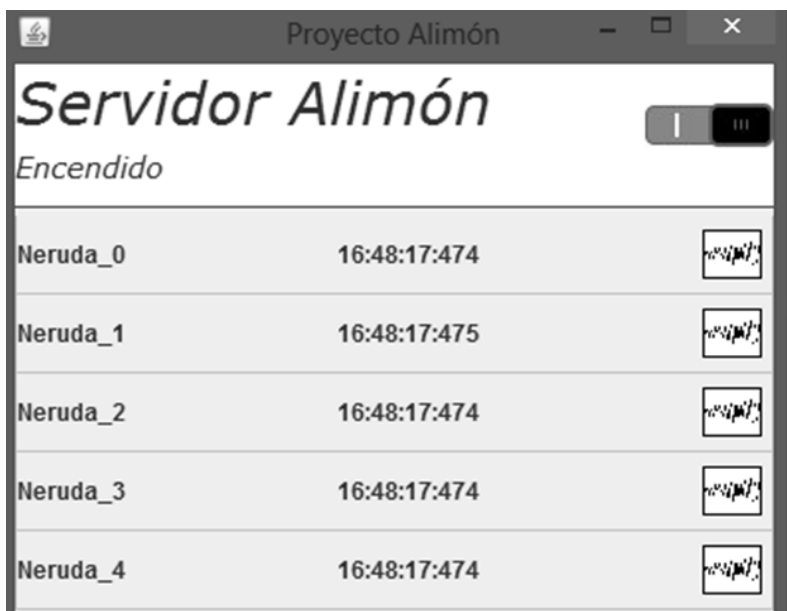

Fig. 6. Imagen de programa para el manejo de conexiones y almacenamiento de datos. 
Para realizar la evaluación del sistema se utilizó un contador como señal de prueba y se realizó la transmisión por 20 minutos. Finalmente se obtuvo la relación de paquetes recibidos con respecto a los paquetes no recibidos, para obtener los paquetes enviados correctamente en cada transmisión. La prueba se repitió 30 veces y se realizó una prueba de normalidad del conjunto de mediciones utilizando la prueba omnibus de D'Agostino-Pearson ( $\mathrm{p}=0.5$ ). Los resultados se presentan en la Tabla 1.

Tabla 1. Tabla de porcentajes de datos recibidos correctamente.

\begin{tabular}{|c|c|}
\hline Número de repeticiones & 30 \\
\hline Tiempo de transmisión & $20 \mathrm{~min}$ \\
\hline Promedio & $99.22 \%$ \\
\hline Desviación Estándar & $0.5996 \%$ \\
\hline
\end{tabular}

Realizando el análisis de normalidad, se concluyó que los porcentajes obtenidos de los paquetes recibidos correctamente corresponden a una distribución gaussiana o normal.

Posteriormente se realizó la prueba de capacidad de conexión máxima del sistema, en la que se simuló la conexión de módulos de adquisición de electroencefalografía enviando datos en forma paralela. El programa "Alimón" tiene capacidad de hasta 12 módulos en paralelo, para no comprometer la visibilidad de la información en la pantalla de despliegue, aunque la cantidad máxima está determinada por las capacidades de la computadora o dispositivo utilizado como servidor. Esta prueba se repitió 10 veces confirmando la viabilidad de la capacidad máxima propuesta con los elementos utilizados.

\section{Discusión}

En este trabajo se obtuvo un sistema mHealth capaz de conectar hasta 12 módulos de registro de señales EEG en forma simultánea a través del protocolo de comunicación Wi-Fi 802.11 b/g con una eficiencia del $99.22 \%$ en la transmisión de la información, lo que implica que llegó ordenada y sin pérdidas. Usar este protocolo permitió realizar una comunicación inalámbrica por medio de redes WLAN de alta calidad, por lo que el ó los pacientes pueden estar en una o varias salas dentro o fuera de la zona donde se ubica el servidor que almacena y analiza la información, siempre y cuando estén dentro del área de cobertura de la red. Esta característica marca la diferencia de este equipo con respecto a los encontrados comercialmente para electroencefalografía, como los mencionados en la sección 1. Este tipo de sistemas mHealth, se han propuesto a nivel experimental para otras señales tales como temperatura [11] y electrocardiografía [18] con el objetivo de probar técnicas de transmisión entre sistemas de registro conectados a computadoras personales y el servidor.

Debido a que el protocolo Wi-Fi es uno de los más usados en forma comercial, el sistema tiene la posibilidad de crear redes con la mayoría de dispositivos existentes en el mercado y presenta varias ventajas en comparación con el protocolo Bluetooth como son: mayor eficiencia, mayor rango de alcance, mayor velocidad de trasmisión, entre otras (ver Tabla 2). Aunado a esto, el pertenecer a la familia de protocolos 
TCP/IP permite enviar datos a través de internet aún fuera del hospital, mediante el uso de sockets. Esto genera la ventaja de poder enviar las señales adquiridas directamente desde el dispositivo a un servidor en internet, sin la necesidad de una PC intermedia.

Tabla 2. Comparación entre protocolos Bluethooth y Wi-Fi (Lee, Su, \& Shen, 2007)

\begin{tabular}{|c|c|c|}
\hline Estándar & Bluethooth & Wi-Fi \\
\hline IEEE Spec. & 802.15 .1 & $802.11 \mathrm{a} / \mathrm{b} / \mathrm{g}$ \\
\hline Banda de Frecuencia & $2.4 \mathrm{GHz}$ & $2.4 \mathrm{GHz}, 5 \mathrm{GHz}$ \\
\hline $\begin{array}{l}\text { Velocidad de } \\
\text { transferencia }\end{array}$ & $1 \mathrm{Mb} / \mathrm{s}$ & $54 \mathrm{Mb} / \mathrm{s}$ \\
\hline Rango nominal & $10 \mathrm{~m}$ & $100 \mathrm{~m}$ \\
\hline $\begin{array}{c}\text { Potencia de transmisión } \\
\text { nominal }\end{array}$ & $0-10 \mathrm{dBm}$ & $15-20 \mathrm{dBm}$ \\
\hline Número de canales de RF & 79 & $14(2.4 \mathrm{GHz})$ \\
\hline Ancho de banda & $1 \mathrm{MHz}$ & $22 \mathrm{MHz}$ \\
\hline Tipo de modulación & GFSK & BPSK, QPSK \\
\hline Cifrado & EQ cifrado de flujo & $\begin{array}{l}\text { RC4 stream cipher } \\
\text { (WEP), } \\
\text { AES block cipher }\end{array}$ \\
\hline Autenticación & secreto compartido & WPA2 \\
\hline Protección de datos & 16-bit CR & 32-bit CRC \\
\hline Coeficiente de eficiencia & 94.41 & 97.18 \\
\hline
\end{tabular}

\section{Conclisión}

Este sistema contribuye en el desarrollo de sistemas mHealth que hacen posible el monitoreo de señales biológicas desde diferentes lugares. La propuesta contempló la generación de un sistema móvil de registro de señal electroencefalográfica con la capacidad de enviar en forma inalámbrica la información a un dispositivo que puede localizarse dentro o fuera de la zona física de registro lo que es una característica indispensable en la generación de sistemas mHealth. Es importante recalcar que algunas patologías, tales como la epilepsia, no son predecibles y que el EEG es el estudio clínico estándar en la identificación de crisis convulsivas, por lo que el registro de esta señal en forma continua sería lo más deseable para la búsqueda de los patrones precursores. Sin embargo, el costo en morbilidad por la atención continua de los pacientes lo hace inviable en el sector público con la infraestructura que se cuenta actualmente. Es por ello que la propuesta plantea la posibilidad de manejar la recepción simultánea de información permitiendo optimizar el uso de la infraestructura disponible en el hospital y en el centro de investigación sobre este tipo de señales.

Por otro lado, la utilización de la transmisión por Wi-Fi garantiza la calidad de la recepción de la señal transmitida que es un requisito técnico indispensable para fines de diagnóstico clínico. 
Otro punto importante es que este sistema propone la creación de archivos de almacenamiento con un formato que maneja la paquetería comercial o de uso libre disponibles con el objetivo de aportar bases de datos para el análisis de esta señal bajo diferentes condiciones. Asimismo, la propuesta plantea la posibilidad de manejar la recepción simultánea de información permitiendo optimizar el uso de la infraestructura disponible en el hospital.

\section{Referencias}

1. S. Sanei y J. Chambers, EEG signal prossesing, Chichester, England.: John Wiley \& Sons Inc., 2007, pp. 10-19.

2. INEGI, «“Estadísticas del Sector Salud y Seguridad Social”,» 2004. [En línea]. Available: http://www.inegi.gob.mx/prod_serv/contenidos/espanol/bvinegi/productos/continuas/social es/salud/2003/cuaderno20a.pdf. [Último acceso: 3007 2014].

3. Ping Yu;Wu, M.X. ; Hui Yu ; Xiao, G.Q., «The Challenges for the Adoption of M-Health,» Service Operations and Logistics, and Informatics, 2006. SOLI '06. IEEE International Conference on, pp. 181 - 186, 2006.

4. Emotiv, «Quick Start Guide \& Maintenance,» 2014. [En línea]. Available: http://emotiv.com/eeg/setup.php. [Último acceso: 0807 2014].

5. V. Venkatesh, S. Rulla, G. S., P. S., M. Munk y V. Venkatesh, «EEG signals are informative for individual cue-response combinations in a visuomotor task.».

6. N. Kannathal, J. Chee, K. Er, K. Lim y O. H. Tat, Chaotic Analysis of Epileptic EEG Signals. In The 15th International Conference on Biomedical Engineering, Springer International Publishing, January, 2014, pp. 652-654.

7. M. Ozbalik, V. Gonzalez-Montoya, S. Gowda y L. Morton, «Limitations of Quantative EEG Analysis in the Adult ICU.,» Neurology,, vol. 82, nº 10, 2014.

8. C. F. Reyes, T. J. Contreras, B. Tovar, L. I. Garay y M. A. Silva, «Detection of absence epileptic seizures using support vector machine.,» de In Electrical Engineering, Computing Science and Automatic Control (CCE), 2013 10th International Conference on, México, 2013.

9. QUASAR USA, «QUASAR USA,» 2014. [En línea]. Available: http://www.quasarusa.com/products_dsi.htm. [Último acceso: 1406 2014].

10. I. Advanced Brain Monitoring, «Advanced Brain Monitoring, Inc.,» 2012. [En línea]. Available: http://www.b-alert.com/. [Último acceso: 2304 2012].

11. R. S. .. Istepanian, A. Sungoor, A. Faisal y N. Philip, «INTERNET OF M-HEALTH THINGS “m-IOT”,» de Conf Proc IEEE Eng Med Biol Soc., London, 2011.

12. P. A., T. J., G. K y M. M., «Designing Mobile Applications to support type 1 diabetes education,» de 11th World Conference on Mobile and Contextual Learning, Helsinki, 2012.

13. E. Kyriacou, C. Pattichis, M. Pattichis, A. Jossif, L. Paraskeva, A. Konstantinides y D. Vogiatzis, «An m-Health Monitoring System for Children,with Suspected Arrhythmias,» de Conference of the IEEE EMBS, Lyon, 2007.

14. Z. Kirtava, T. Gegenava y M. Gegenava, «mHealth for Cardiac Patients Telemonitoring and Integrated Care,» de IEEE HEALTHCOM 2013 - The 1st International Workshop on Service Science for e-Health, Portugal, 2013. 
15. A. M. Patel, P. K. Gakare y A. N. Cheeran, «Real Time ECG Feature Extraction and Arrhythmia Detection on a Mobile Platform,» International Journal of Computer Applications, vol. 44, $\mathrm{n}^{\mathrm{o}}$ 23, pp. 40-45, 2012.

16. R. Istepanian, N. Philip, X. H. Wang y S. Laxminarayan, «Non-telephone Healthcare: The Role of 4G and Emerging Mobile Systems for Future m-Health Systems,» Communications in Medical and Care Compunetics, vol. 2, pp. 9-16, 2011.

17. C. f. Reyes, Sistema móvil para adquisición y transmisión de la señal EEG asistido con realidad aumentada para auxiliar el diagnóstico clínico, Mexico, D.F: SEPI-UPIITA-IPN, 2014.

18. E. Kyriacou, C. Pattichis, M. Pattichis, A. Jossif, L. Paraskeva, A. Konstantinides y D. Vogiatzis, «An m-Health Monitoring System for Children,» de Conference of the IEEE EMBS, Lyon, 2007.

19. J.-S. Lee, Y.-W. Su y C.-C. Shen, «A Comparative Study of Wireless Protocols: Bluetooth, UWB, ZigBee, and Wi-Fi,» de The 33rd Annual Conference of the IEEE Industrial Electronics Society (IECON), Taipei, Taiwan, 2007. 